\title{
A case-control study of the impact of the East Anglian breast screening programme on breast cancer mortality
}

\author{
PC Allgood ${ }^{*, 1}$, J Warwick', RML Warren ${ }^{2}$, NE Day ${ }^{3}$ and SW Duffy' \\ 'Cancer Research UK Department of Epidemiology, Mathematics and Statistics, Wolfson Institute of Preventive Medicine, Charterhouse Square, London \\ ECIM 6BQ, UK; ${ }^{2}$ Department of Radiology, Addenbrooke's Hospital, Cambridge CB2 2QQ, UK; ${ }^{3}$ Strangeways Research Laboratory, Wort's Causeway, \\ Cambridge CBI 8RN, UK
}

\begin{abstract}
Although breast cancer screening has been shown to work in randomised trials, there is a need to evaluate service screening programmes to ensure that they are delivering the benefit indicated by the trials. We carried out a case-control study to investigate the effect of mammography service screening, in the NHS breast screening programme, on breast cancer mortality in the East Anglian region of the UK. Cases were deaths from breast cancer in women diagnosed between the ages of 50 and 70 years, following the instigation of the East Anglia Breast Screening Programme in 1989. The controls were women (two per case) who had not died of breast cancer, from the same area, matched by date of birth to the cases. Each control was known to be alive at the time of death of her matched case. All women were known to the breast screening programme and were invited, at least once, to be screened. There were 284 cases and 568 controls. The odds ratio (OR) for risk of death from breast cancer in women who attended at least one routine screen compared to those who did not attend was 0.35 ( $\mathrm{Cl}: 0.24,0.50)$. Adjusting for self-selection bias gave an estimate of the breast cancer mortality reduction associated with invitation to screening of $35 \%(\mathrm{OR}=0.65,95 \% \mathrm{Cl}: 0.48,0.88)$. The effect of actually being screened was a $48 \%$ breast cancer mortality reduction $(\mathrm{OR}=0.52,95 \% \mathrm{Cl}: 0.32,0.84)$. The results suggest that the National Breast Screening Programme in East Anglia is achieving a reduction in breast cancer deaths, which is at least consistent with the results from the randomised controlled trials of mammographic screening.

British Journal of Cancer (2008) 98, 206-209. doi: I0.1038/sj.bjc.6604I23 www.bjcancer.com
\end{abstract}

Published online 4 December 2007

(c) 2008 Cancer Research UK

Keywords: breast cancer mortality; screening; case-control

Randomised trials have shown that breast screening reduces breast cancer mortality (Jonsson et al, 2000, 2001, 2003; Duffy et al, 2002; Nyström et al, 2002; Tabar et al, 2003). Whether this is achieved in routine practice requires monitoring as certain factors might alter the effect. These include technical changes in the mammographic equipment since the trials, different screening frequencies to those of the trials, expertise of the professionals involved, and quality of the assessment process. For this reason, quality assurance is a fundamental part of the National Health Service Breast Screening Programme (NHSBSP), which aims at minimum standards and improved performance so that women have access to high-quality screening service wherever they live (NHS, 2006).

The case-control design is convenient and its basic principle here is simple: women who have died from the disease are selected as the cases and for each, we select as controls a number of agematched women (usually one or two) known to be alive at the time of death of the case. We then compare the screening histories of cases and controls prior to the diagnosis dates of the cases. If the screening is reducing mortality from the disease, the cases will tend to have lesser screening histories than the controls.

The design is prone to certain biases, in particular, self-selection bias, since women who accept the invitation to screening may have

*Correspondence: Dr PC Allgood; E-mail: prue.allgood@cancer.org.uk Received 24 August 2007; revised 2 November 2007; accepted 5 November 2007; published online 4 December 2007 better health status a priori than those who do not, conferring a reduced likelihood of dying from the disease independent of screening. This is a bias in favour of screening, and a method for correcting it is given by Duffy et al (2002).

We therefore conducted a case-control study to

(i) estimate the effect of the NHSBSP on breast cancer mortality in the East Anglian region of the UK, adjusting for selfselection bias; and

(ii) ascertain whether the reduction in breast cancer mortality being achieved is consistent with that observed in the randomised screening trials.

\section{MATERIALS AND METHODS}

All female residents of the East Anglian region (as delineated when screening began) aged 50-70 years and registered with a general practitioner were invited to attend breast screening once in every 3 years. The NHSBSP was established in the counties of Norfolk, Suffolk, and Cambridgeshire in 1989.

For sample size, assuming a relative risk of 0.68 (Cuzick et al, 1997), and a 70\% coverage, 264 cases and 528 matched controls would detect a significant effect with $80 \%$ power at the $5 \%$ level with a two-sided test (Breslow and Day, 1987). We therefore aimed for 300 cases and 600 controls as a fail-safe measure. Cases were deaths from female breast cancer in women aged 50-70 years at 
diagnosis from 1995 onwards, to avoid the accumulation found in the prevalent round of the screening programme, and to minimise screening opportunity bias (Walter, 2003). Deaths were included for which breast cancer was given as the primary or a contributory cause on the death certificate.

We excluded those women who had not been invited to breast screening at least once. Cases were taken at random from the EA cancer registry database. There were two controls for each case and matched by date of birth, most coming from the same health authority. The controls were all alive at the time of death of their case and were also known to the data source before the diagnosis of the case. Controls were obtained from the National Health Service (NHS) Exeter System database and individual screening units. Each control was allocated a pseudodiagnosis date equal to the date of diagnosis of that control's matched case. The following information was obtained:

- date of birth,

- date of diagnosis (if a case),

- stage at diagnosis (if a case),

- date of death (if a case), and

- complete screening history including dates of invitations and attendances, and outcomes of attendances.

\section{Statistical analysis}

The data were analysed by conditional logistic regression. The primary outcome of whether a woman had ever attended breast screening prior to diagnosis/pseudodiagnosis is potentially subject to self-selection or healthy volunteer bias, as it compares women who choose to take up the invitation to screening with women who do not. The latter may have poorer health $a$ priori and therefore be more likely to die of the disease, independent of screening. An adjustment can be made for this using the relative rate of breast cancer deaths in non-attenders in the randomised trials compared to the trial controls (Duffy et al, 2002).

As an alternative, we adjusted for socio-economic status as measured by the index of multiple deprivation (IMD) derived from the womens' postcodes, in the logistic regression, as this is the main confounding factor of outcome and attendance. The IMD is an area-based measure of relative deprivation, based on census statistics for overcrowded housing and other factors. Secondary analyses included the time since last screen attended, age at first attendance, and the number of screens attended.

\section{RESULTS}

Out of the 300 cases identified, 16 were excluded because they were diagnosed while awaiting their first screening invitation. The remaining 284 were matched on birth date to two controls each, giving a total study population of 852 women.

Slightly more controls than cases were in the two most affluent deprivation groups 1 and 2 (51 versus 48\%) and slightly fewer in groups 3 and 4 , but this was not significant $(P=0.3)$. The average age at diagnosis/pseudodiagnosis was 60.7 years (range $50.4-70.5$ ) for both cases and controls as expected due to the matching. Mean age at first screen was 55.3 for cases and 55.5 for controls (Table 1). The mean number of screens was 1.39 for the cases compared with 1.70 for the controls, a significant difference $(P<0.001)$.

More than $85 \%(242 / 284)$ of the cases presented symptomatically, $58 \%$ as interval cancers and $27 \%$ in women who had never attended screening. As might be expected with fatal cancers, the tumours were at relatively advanced stages, with $49 \%$ being at TMN stage 2 and $37 \%$ at stage 3 or 4 . The average age at death was 63.0 years (range $50.8-77.6$ ) and average time from diagnosis to death was 2.4 years, ranging from 3 days to 8.8 years. The years of diagnosis and death ranged from 1995 to 2004.
Table I Patient characteristics, screening history by case-control status

\begin{tabular}{|c|c|c|}
\hline & Cases & Controls \\
\hline Total numbers & 284 & 568 \\
\hline \multicolumn{3}{|l|}{ Index of multiple deprivation (IMD), N (\%) ${ }^{\mathrm{a}}$} \\
\hline Ist quartile & $75(26)$ & $135(25)$ \\
\hline 2nd quartile & $61(22)$ & $146(26)$ \\
\hline 3rd quartile & $68(24)$ & $139(25)$ \\
\hline 4th quartile & $80(28)$ & $132(24)$ \\
\hline $\begin{array}{l}\text { Mean age at diagnosis or pseudodiagnosis } \\
\text { (range) }\end{array}$ & \multicolumn{2}{|c|}{$60.7(50.4-70.5) 60.7(50.4-70.5)$} \\
\hline \multicolumn{3}{|l|}{ Age group, $N(\%)$} \\
\hline $50-54$ & $50(18)$ & $100(18)$ \\
\hline $55-59$ & $85(29)$ & $170(29)$ \\
\hline $60-64$ & $67(24)$ & $134(24)$ \\
\hline $65+$ & $82(29)$ & $164(29)$ \\
\hline Mean age at first screen, years (range) & \multicolumn{2}{|c|}{$55.3(45.2-65.4) 55.5(43.5-65.5)$} \\
\hline \multicolumn{3}{|l|}{ Number of screening visits, $N(\%)$} \\
\hline 0 & $77(27)$ & $63(11)$ \\
\hline । & $87(31)$ & $185(32)$ \\
\hline 2 & $66(23)$ & $198(35)$ \\
\hline 3 & $45(16)$ & $106(19)$ \\
\hline $4+$ & $10(3)$ & $16(3)$ \\
\hline Mean number of screens (range) & $1.39(0-5)$ & $1.70(0-5)$ \\
\hline \multicolumn{3}{|l|}{ Outcome of first screen, $N$ (\%) } \\
\hline Routine recall & $190(92)$ & $498(99)$ \\
\hline Recall to assessment and benign & $4(2)$ & $7(1)$ \\
\hline Recall to assessment and cancer & $13(6)$ & $0(0)$ \\
\hline \multicolumn{3}{|l|}{ Mode of detection, $N(\%)$} \\
\hline Non-attender & $77(27)$ & \\
\hline First screen (screen detected) & $10(3)$ & \\
\hline Subsequent screen (screen detected) & $32(12)$ & \\
\hline Interval cancer & $165(58)$ & \\
\hline \multicolumn{3}{|l|}{ TMN stage, $N(\%)^{b}$} \\
\hline 1 & $37(14)$ & \\
\hline 2 & $137(49)$ & \\
\hline 3 & $25(9)$ & \\
\hline 4 & $76(28)$ & \\
\hline Mean age at death, years (range) & $63.0(50.8-7$ & \\
\hline
\end{tabular}

The unadjusted OR of women who attended at least one routine screen compared to those who never attended screening was 0.35 (95\% CI: $0.24,0.51 ; P<0.001$ ) (Table 2). Adjusting for selfselection bias using the more conservative intention-to-treat analysis (Duffy et al, 2002) gave a $35 \%$ estimated mortality reduction $(\mathrm{OR}=0.65,95 \% \mathrm{CI}: 0.48,0.88 ; P=0.005)$. Actually being screened, adjusted for self-selection bias, was associated with a $48 \%$ reduction $(\mathrm{OR}=0.52,95 \%$ CI $0.32,0.84 ; P=0.007)$. As an alternative to self-selection bias correction, adjustment for IMD had no effect on the OR associated with ever being screened $(\mathrm{OR}=0.35,95 \%$ CI $0.23-0.51, P<0.001)$.

The major effect of any screens attended is an approximately $60 \%$ lower risk of death from breast cancer compared to those who had attended none; there was no significant effect for number of screens attended. Risk was higher in those with a screen within 1 year than those whose last screen was between 1 and 2 years prior to diagnosis or pseudodiagnosis, due to deaths from screendetected cancers. Thereafter, risk increased with increasing time since last screen. Risk was higher in those whose first screen took 
Table 2 Odds ratios for risk of death from breast cancer by screening history 1995-2004

\begin{tabular}{|c|c|c|}
\hline Screening factors & $\begin{array}{l}\text { No. of cases/ } \\
\text { controls }\end{array}$ & $\begin{array}{l}\text { Odds ratio } \\
\text { (95\% CI) }\end{array}$ \\
\hline \multicolumn{3}{|l|}{ Ever attended } \\
\hline Never & $77 / 63$ & I \\
\hline Ever & $207 / 505$ & $0.35(0.24,0.50)$ \\
\hline Self-selection corrected (invitation effect) & & $0.65(0.48,0.88)$ \\
\hline Self-selection corrected (screening effect) & & $0.52(0.32,0.84)$ \\
\hline \multicolumn{3}{|l|}{ Number of screens attended } \\
\hline I & $86 / 185$ & l \\
\hline 2 & $66 / 198$ & $0.70(0.43,1.11)$ \\
\hline $3+$ & $55 / 122$ & $1.03(0.59,1.77)$ \\
\hline None & $77 / 63$ & $2.51(1.56,4.03)$ \\
\hline \multicolumn{3}{|l|}{ Time since last screen } \\
\hline$<$ I year & $65 / 113$ & 1 \\
\hline $1<2$ years & $4 \mid / 121$ & $0.43(0.24,0.77)$ \\
\hline $2<4$ years & $62 / 175$ & $0.48(0.28,0.81)$ \\
\hline $4+$ years & $39 / 96$ & $0.55(0.29,1.04)$ \\
\hline Never screened & $77 / 63$ & $1.71(1.03,2.80)$ \\
\hline \multicolumn{3}{|l|}{ Age at first screen (screened women only) } \\
\hline$<52$ & $53 / 144$ & 1 \\
\hline $52-53$ & $50 / 91$ & $2.38(1.02,5.57)$ \\
\hline $54<58$ & $60 / 137$ & $2.25(0.83,6.09)$ \\
\hline$>58$ & $44 / 133$ & $1.54(0.33,7.01)$ \\
\hline
\end{tabular}

place at age 52 or more compared to age 51 or less, but the trend was not significant, possibly due to the small numbers.

\section{DISCUSSION}

The results of this study are encouraging. After adjusting for selfselection bias, we found a significant $35 \%$ reduction in breast cancer mortality in association with invitation to screening and a significant $48 \%$ reduction with actually being screened, if anything, exceeding the benefits in the randomised trials (Smith et al, 2004). However, the correction method makes an important assumption that the relative mortality for non-compliers compared with a population not invited for screening is the same in the programme in question as in the randomised trials. From the available information (the Swedish Two-county, Malmö, Gothenburg, Stockholm and Canadian trials) (Duffy et al, 2002), non-attenders had a $36 \%$ higher risk of breast cancer mortality than uninvited controls. This may not hold for our population, but it seems likely that there is at least some self-selection bias, and the uncorrected estimates of the mortality reduction seem implausible.

\section{REFERENCES}

Breslow NE, Day NE (1987) Statistical Methods in Cancer Research Volume II- The Design and Analysis of Cohort Studies. Lyon: International Agency for Research on Cancer

Cuzick J, Edwards R, Segnan N (1997) Adjusting for non-compliance and contamination in randomized screening trials. Stat Med 16: $1017-1029$

Duffy SW, Cuzick J, Tabar L, Vitak B, Hsiu-Hsi Chen T, Yen M-F, Smith RA (2002) Correcting for non-compliance bias in case-control studies to evaluate cancer screening programmes. Appl Statist 51: 235-243

Elmore JG, Reisch LM, Barton MB, Barlow WE, Rolnick S, Harris EL, Herrinton LJ, Geiger AM, Beverly RK, Hart G, Yu O, Greene SM, Weiss NS, Fletcher SW (2005) Efficacy of breast cancer screening in the community according to risk level. J Natl Cancer Inst 97: 1035-1043

Fielder HM, Warwick J, Brook D, Gower-Thomas K, Cuzick J, Monypenny I, Duffy SW (2004) A case-control study to estimate the impact on
There is a suggestive increase in risk with first screen at 52 or over, suggesting that there is an advantage to a first screen at the lower age limit of 50, though the trend was not significant; it should be examined in other studies.

Adjustment for IMD made no difference to the estimated effect of screening on risk, perhaps because although socioeconomic status is strongly associated with screening in urban areas, it may not be so in rural areas. In East Anglia, IMD was not strongly related to screening exposure, around $84 \%$ regardless of IMD.

There was no substantial trend in risk with number of screens attended, consistent with the fact that only the screen at which a cancer is detected actually confers a benefit (Elmore et al, 2005). Aside from a high risk in those very recently screened, there was an increase in the risk with time since last screen. The high risk in those screened in the last year could be due to screen-detected cancers. When these are excluded, the ORs for 1-2,2-4, and 4 or more years since last screen, relative to less than 1 year, were 1.05 , 1.32 , and 1.49 respectively.

From other case-control screening studies, there is a range of results from a very small benefit to large reductions in mortality as observed in this study (Palli et al, 1989; Mittenburg et al, 1998; Fielder et al, 2004; Elmore et al, 2005; Gabe et al, 2007). Our estimated benefit may be slightly biased in favour of screening by the selection of recently diagnosed cases (1995-2004), which will of necessity be relatively rapidly fatal and therefore may underrepresent screening-exposed fatal cancers. Restricting analysis to those diagnosed before 1999, we obtain an uncorrected mortality reduction of $59 \%$ with ever being screened, and self-selection bias corrected estimates of $27 \%$ for invitation to screening, and $37 \%$ for actually being screened.

In conclusion, this study shows strong evidence that the NHS breast screening programme in East Anglia is achieving at least a $30 \%$ reduction in breast cancer mortality in women attending screening, consistent with the randomised trials. We recommend expanding the case-control study to other regions of the UK and internationally.

\section{ACKNOWLEDGEMENTS}

We would like to thank Sue Halliday at the East of England Quality Assurance Reference Centre for allowing us to carry out the study; Sara Godward, Karen Wright, and David Greenberg at the Cancer Intelligence Unit (formerly the East Anglian Cancer Registry) for supplying the cases, breast cancer mortality statistics and answering queries; Paul Smith at the Eastern Region Public Health Observatory for supplying us with the Index of Multiple Deprivation Score; and especially the directors and all the staff at the individual breast screening units in East Anglia for allowing us to use their data and answering numerous queries. breast cancer death of the breast screening programme in Wales. J Med Screen 11: 194-198

Gabe R, Tryggvadóttir L, Sigfússon BF, Ólafsdóttir GH, Sigurðsson K, Duffy SW (2007) A case-control study to estimate the impact of the Icelandic population-based mammography screening programme on breast cancer death. Acta Radiol (in press)

Jonsson H, Nyström L, Törnberg S, Lenner P (2001) Service screening with mammography of women aged 50-69 years in Sweden: effects on mortality from breast cancer. J Med Screen 8: 152-160

Jonsson H, Nyström L, Törnberg S, Lundgren B, Lenner P (2003) Service screening with mammography. Long-term effects on breast cancer mortality in the county of Gävleborg, Sweden. The Breast 12: 183-193

Jonsson H, Törnberg S, Nyström L, Lenner P (2000) Service screening with mammography in Sweden. Evaluation of effects from screening 
in age 40-49 years on the breast cancer mortality. Acta Oncol 39: $617-623$

Mittenburg GAJ, Peeters PHM, Fracheboud J, Collette HJA (1998) Seventeen-year evaluation of breast cancer screening: the DOM project, The Netherlands. Br J Cancer 78: $962-965$

NHS Breast Screening Programme (2006) Annual Review 2005. NHS: Sheffield Nyström L, Andersson I, Bjurstam N, Frisell J, Nordenskjöld B, Rutqvist LE (2002) Long-term effects of mammography screening: updated overview of the Swedish randomised trials. Lancet 359: 909-919

Palli D, Rosselli del Turco M, Uiatti E, Ciatto S, Crocetti E, Paci E (1989) Time interval since last test in a breast cancer screening programme: a case-control study in Italy. J Epidemiol Community Health 43: $241-248$

Smith RA, Duffy SW, Gabe R, Tabar L, Yen AMF, Chen THH (2004) The randomized trials of breast cancer screening: what have we learned? Radiol Clin N Am 42: 793-806

Tabar L, Yen MF, Vitak B, Chen HH, Smith RA, Duffy SW (2003) Mammography service screening and mortality among breast cancer cases: results of 20-year follow-up before and after introduction of screening. Lancet 361: $1405-1410$

Walter SD (2003) Mammographic screening: case-control studies. Ann Oncol 14: $1190-1192$ 